\title{
Bioengineering of Improved Biomaterials Coatings for Extracorporeal Circulation Requires Extended Observation of Blood-Biomaterial Interaction under Flow
}

\author{
Kris N. J. Stevens, ${ }^{1}$ Yvette B. J. Aldenhoff, ${ }^{2}$ Frederik H. van der Veen, ${ }^{1}$ Jos G. Maessen, ${ }^{1}$ and Leo H. Koole ${ }^{1}$ \\ ${ }^{1}$ Department of Cardiothoracic Surgery, Academic Hospital Maastricht, P.O. Box 5800, 6200 MD Maastricht, The Netherlands \\ ${ }^{2}$ Centre for Biomaterials Research, University of Maastricht, P.O. Box 616, 6200 MD Maastricht, The Netherlands
}

Correspondence should be addressed to Leo H. Koole, 1.koole@bioch.unimaas.nl

Received 3 April 2007; Revised 4 July 2007; Accepted 3 December 2007

Recommended by John L. McGregor

Extended use of cardiopulmonary bypass (CPB) systems is often hampered by thrombus formation and infection. Part of these problems relates to imperfect hemocompatibility of the $\mathrm{CPB}$ circuitry. The engineering of biomaterial surfaces with genuine longterm hemocompatibility is essentially virgin territory in biomaterials science. For example, most experiments with the well-known Chandler loop model, for evaluation of blood-biomaterial interactions under flow, have been described for a maximum duration of 2 hours only. This study reports a systematic evaluation of two commercial CPB tubings, each with a hemocompatible coating, and one uncoated control. The experiments comprised (i) testing over 5 hours under flow, with human whole blood from 4 different donors; (ii) measurement of essential blood parameters of hemocompatibility; (iii) analysis of the luminal surfaces by scanning electron microscopy and thrombin generation time measurements. The dataset indicated differences in hemocompatibility of the tubings. Furthermore, it appeared that discrimination between biomaterial coatings can be made only after several hours of bloodbiomaterial contact. Platelet counting, myeloperoxidase quantification, and scanning electron microscopy proved to be the most useful methods. These findings are believed to be relevant with respect to the bioengineering of extracorporeal devices that should function in contact with blood for extended time.

Copyright (c) 2007 Kris N. J. Stevens et al. This is an open access article distributed under the Creative Commons Attribution License, which permits unrestricted use, distribution, and reproduction in any medium, provided the original work is properly cited.

\section{INTRODUCTION}

Cardiopulmonary bypass $(\mathrm{CPB})$ technology represents one of the most striking examples of progress in biomedical engineering. Procedures in cardiac surgery that rely on $\mathrm{CPB}$ can nowadays be regarded as safe, that is, they are associated with a low incidence of mortality [1-3]. These developments resulted, to a significant extent, from improvements in the polymeric biomaterials that constitute the inner surface of CPB circuits. Within CPB circuits, there is extensive blood contact with the tubing, the pump, and, particularly, the oxygen/carbon dioxide exchange membrane. It is well known that cellular components of the blood (particularly, leukocytes and platelets) may become activated, and that four different but partially overlapping plasma protein cascades will go into operation (intrinsic and extrinsic clotting cascade, complement system, and fibrinolytic protein system)
[4]. For more than 4 decades, heparin has been used as the standard "anticoagulant" to counterbalance these effects. Improvements resulted from the use of surface coatings exposing heparin at the blood-biomaterial interface. These coatings reduce coagulation, inflammation, complement activation, and platelet activation [2, 4]. More recently, CPB equipment has been coated with poly(2-methoxy-ethylacrylate) (PMEA), based on the hypothesis that this material leads to improved blood compatibility compared to uncoated surfaces. PMEA is cheap compared to heparin-exposing coatings and was postulated to provide a useful alternative for patients with heparin-associated disorders [5-7].

However, serious complications may arise if extracorporeal circulation has to be sustained, that is, for several days. The most frequent problems stem from bacterial infection, hemolysis, thrombus formation within the circuit, and formation of circulating thrombotic emboli [8-10]. We became 
intrigued by these problems, since they relate to long-term hemocompatibility of polymeric materials, which is in fact unexplored territory in biomaterials science. Indeed, we noticed that the literature on blood compatibility of CPB circuits merely contains experimental data that correspond to short testing periods. For example, Weber et al. extensively studied hemocompatibility of 4 different biomaterials in a CPB model, but only up to 120 minutes [11]. We adhere to the idea that successful development of novel biomaterials or biomaterial coatings for CPB will depend on robust evaluation models in which the blood-biomaterial contact is maintained for several hours at least.

Herein we report a systematic methodological study in which two commercial surface-coated $\mathrm{CPB}$ tubings (heparin-coated tubings and PMEA-coated tubings) and one uncoated control were evaluated in contact with human whole blood under flow, for a period of 5 hours. We calculated that 5 hours of experimentation implies a level of blood-biomaterial contact that corresponds to at least 9 hours of operation in a typical CPB system (vide infra). Several assays were used to evaluate the blood (platelet counts and assays to determine hemolysis, platelet activation, leukocyte activation, and activation of the complement system). Scanning electron microscopy (SEM) was used to study deposition of blood components at the surface of the tubings. A full set of data was acquired for the three different materials, four donors and five time points $(0,75,150,225$, and 300 minute). The three materials showed clear differences, in general pointing towards an inferior hemocompatibility of the PMEA coating. Moreover, two points with respect to bioengineering of improved coatings for long-term $\mathrm{CPB}$ emerged as follows: (i) since most of the differences between the three surfaces did not become apparent during the first 2 hours of experimentation, long-term (e.g., 5 hour) testing of blood-biomaterial interactions under flow is required; (ii) preferably, parallel tests with blood from several different donors should be performed, since the results from several assays appeared to be clearly donor-dependent.

\section{MATERIALS AND METHODS}

\subsection{Materials}

Polyvinyl chloride (PVC) tubings with a coating of PMEA were a generous gift of Terumo Europe NV (Leuven, Belgium). The internal diameter of the tubings was $0.476 \mathrm{~cm}$. The same company also provided the uncoated tubings with identical internal diameter, which were used as controls. Tubings with a coating of heparin were obtained from Maquet Cardiopulmonary AG (Hirrlingen, Germany). The internal diameter was also $0.476 \mathrm{~cm}$. All tubings were received in a sterile package and cut to length of $(42.5 \mathrm{~cm})$ immediately prior to the experiments. Lepirudin (Refludan) was purchased from Pharmion (Windsor Berkshire, UK). Bovine serum albumin (BSA), Na-citrate, ethylenediaminetetraacetic acid (EDTA), and Zymosan A were from Sigma-Aldrich Chemie B.V. (Zwijndrecht, The Netherlands). 4-(2-hydroxyethyl)-1-piperazineethanesulfonic acid (HEPES), $\mathrm{NaCl}, \mathrm{KCl}$, and Glutaraldehyde $25 \%$ were from
Acros Organics (Geel, Belgium). $\mathrm{Na}_{2} \mathrm{HPO}_{4}$ and $\mathrm{KH}_{2} \mathrm{PO}_{4}$ were from Janssen Chimica (Beerse, Belgium). Ethanol 100\% was from Merck KGaA (Darmstadt, Germany). The chromogenic substrate S2238 was synthesized according to $\mathrm{Ri}$ jkers et al. [12]. The following solutions were prepared: a lepirudin stock solution (lepirudin $200 \mu \mathrm{g} / \mathrm{mL}, \mathrm{NaCl} 9 \mathrm{~g} / \mathrm{L}$ ), a HEPES/EDTA stock solution (HEPES $100 \mathrm{mM}$, EDTA $40 \mathrm{mM}, \mathrm{pH} 7.4$ ), a phosphate-buffered saline (PBS) solution $\left(\mathrm{NaCl} 8 \mathrm{~g} / \mathrm{L}, \mathrm{KCl} 0.2 \mathrm{~g} / \mathrm{L}, \mathrm{Na}_{2} \mathrm{HPO}_{4} 1.44 \mathrm{~g} / \mathrm{L}, \mathrm{KH}_{2} \mathrm{PO}_{4}\right.$ $0.24 \mathrm{~g} / \mathrm{L}, \mathrm{pH} 7.4)$, a $\mathrm{CaCl}_{2}$ stock solution $\left(0.5 \mathrm{M} \mathrm{CaCl}_{2}\right)$, a $\mathrm{Na}$-citrate stock solution (Na-citrate $0.13 \mathrm{M}$ ), an S2238 stock solution (S2238 $2 \mathrm{mM}$ ), and a stop buffer $(\mathrm{NaCl} 140 \mathrm{mM}$, HEPES $20 \mathrm{mM}$, EDTA $20 \mathrm{mM}$, BSA $1 \mathrm{mg} / \mathrm{mL}$, S2238 stock solution 1/10, $\mathrm{pH}$ 7.5). Citrate, theophylline, adenosine, dipyridamole (CTAD) stock solution (BD Vacutainer CTAD Tubes) was a product from Becton Dickinson (Alphen aan den Rijn, The Netherlands). The enzyme-linked immunosorbent assay (ELISA) for $\beta$-thromboglobulin ( $\beta$-TG) (Asserachrom $\beta$-TG) was purchased from Diagnostica Stago (Asnières sur Seine, France) and ELISA kits for terminal complement complex (TCC) and myeloperoxidase (MPO) were from Hycult biotechnology B.V. (Uden, The Netherlands).

\subsection{Equipment}

Experiments were performed on a modified Chandler loop system, which was equipped with a broad wheel with a diameter of $13 \mathrm{~cm}$ [13]. On this wheel, 12 tubes could be rotated simultaneously. The rotating speed was set at 32 per minute. The rotating wheel and the mounted tubes were immersed in a water bath that was kept at $37^{\circ} \mathrm{C}$ throughout the entire experiment. The Chandler loop device was made by the mechanical workshop of the Instrument Development Engineering \& Evaluation of the University Maastricht. Centrifugation was performed with an Eppendorf Centrifuge 5417C (Eppendorf, Hamburg, Germany). Platelets were counted on an automatic cell counter (Coulter AC-T diff, Beckman Coulter, Miami, Fl, USA). The absorbance of plasmafree hemoglobin $(\mathrm{Hb})$ was determined on a spectrophotometer (Multiskan Spectrum Microplate Spectrophotometer, Thermo Labsystems, Vantaa, Finland). Microtiter plates were heated on a plate warmer (Single Micro-Hywel, Chromogenix, Milano, Italy). For both the ELISA assays and the thrombin generation time assay, the absorbances of the microtiter plates were determined spectrophotometrically on a microplate reader (ELx808 Absorbance Microplate Reader, BioTek Instruments, Inc., Vt, USA). Samples for SEM were coated with gold on a sputter coater (Sputter coater 108 auto/SE, Cressington Scientific Instruments Ltd., Watford, $\mathrm{UK}$ ) and then analyzed with a scanning electron microscope (Philips XL30 Scanning Electron Microscope, Philips, Eindhoven, The Netherlands).

\subsection{Experiments under flow conditions: the Chandler loop model}

This study was approved by the Ethical Committee of the University of Maastricht. Four healthy male blood donors 
(further indicated by their initials as WW, KS, SB, and JB) aged between 20 and 25 years old were included in this study. They were all nonsmokers and did not take any haemostasisinfluencing medicines at least 10 days before the experiment. Each donor visited our laboratories twice and donated blood for two different experiments; there were at least 7 days between the two visits.

\subsubsection{Hemocompatibility analysis by platelet counting and assessment of hemolysis (performed after the first visit of each donor)}

Blood was withdrawn by venipuncture and immediately anticoagulated with lepirudin stock solution (1 part lepirudin stock solution and 9 parts whole blood), following recommendations made by Kopp et al. [14]. Directly after blood collection, $1.35 \mathrm{~mL}$ of blood was sampled and processed as described further to obtain baseline values. Next, three different tubes (one heparin-coated tube, one PMEA-coated tube, and one uncoated control tube) were each filled with $6.7 \mathrm{~mL}$ whole blood, which corresponds to a degree of filling of $88 \%$. The tubes were then closed end-to-end using silicon sleeves, mounted on the rotating wheel, and rotated in a water bath at $37^{\circ} \mathrm{C}$ and $32 \mathrm{rpm}$.

From each tube, $1.35 \mathrm{~mL}$ blood was withdrawn after 75, 150,225 , and 300 minutes of incubation; note that in the tubes, the degree of filling gradually dropped from $88 \%$, via $70 \%$ and $53 \%$, to $35 \%$. Immediately after withdrawal from the tube, each blood sample was mixed with $150 \mu \mathrm{L}$ HEPES/EDTA stock solution. One third of this mixture $(500 \mu \mathrm{L})$ was used for platelet counting; these counts were performed intriplicate.

The other part of the HEPES/EDTA-mixed blood sample $(1 \mathrm{~mL})$ was processed for assessment of hemolysis. The percentage of plasma-free $\mathrm{Hb}$ was used as an indicator for hemolysis. $25 \mu \mathrm{L}$ of HEPES/EDTA-mixed blood sample was diluted 40 times with $975 \mu \mathrm{L}$ deionized water to achieve total hemolysis. The other $975 \mu \mathrm{L}$ of HEPES/EDTA-mixed blood sample was kept undiluted. Both the diluted and undiluted parts were centrifuged $\left(3220 \mathrm{~g}, 20\right.$ minutes, $\left.4^{\circ} \mathrm{C}\right)$ to obtain plasma. Subsequently, the absorbance was measured at three wavelengths $(560,576$, and $592 \mathrm{~nm})$ in plasma of both the diluted and undiluted parts of the HEPES/EDTA-mixed blood sample. The percentage of plasma-free $\mathrm{Hb}$ was then calculated for each blood sample according to the procedure of Cripps [15].

\subsubsection{Hemocompatibility analysis by quantification of blood activation markers via ELISA and SEM of the tube inner surfaces (performed after the second visit of each donor)}

Blood was withdrawn by venipuncture and immediately anticoagulated with lepirudin stock solution (1 part lepirudin stock solution and 9 parts whole blood), following recommendations made by Kopp et al. [14]. Immediately after blood collection, a $4.5 \mathrm{~mL}$ and $1.8 \mathrm{~mL}$ blood sample were isolated and processed as described further to obtain baseline values. Next, twelve tubes (four heparin-coated tubes, four
PMEA-coated tubes, and four uncoated control tubes) were each filled with $6.7 \mathrm{~mL}$ whole blood, which corresponds to a degree of filling of $88 \%$. The tubes were then closed endto-end using silicon sleeves, mounted on the rotating wheel, and rotated in a water bath at $37^{\circ} \mathrm{C}$ and $32 \mathrm{rpm}$.

After 75, 150, 225, and 300 minutes of incubation, each time three tubes (one heparin-coated tube, one PMEAcoated tube and, an one uncoated control tube) were removed from the rotating wheel. Two blood samples were isolated from each tube: $4.5 \mathrm{~mL}$ blood was withdrawn and immediately mixed with $0.5 \mathrm{~mL}$ CTAD stock solution, and $1.8 \mathrm{~mL}$ of blood was withdrawn and immediately mixed with $0.2 \mathrm{~mL}$ HEPES/EDTA stock solution. Both the CTAD-mixed and HEPES/EDTA-mixed blood were incubated on ice for 15 minutes. Then, plasma was isolated by two subsequent centrifugation steps $\left(2550 \mathrm{~g}, 20\right.$ minutes, $\left.4^{\circ} \mathrm{C}\right)$. The plasma was aliquoted and stored at $-80^{\circ} \mathrm{C}$ until further analysis. ELISA was used to evaluate activation of leukocytes, complement, and platelets. As a marker for leukocyte activation the levels of MPO were quantified in HEPES/EDTAstabilized plasma. The concentration of $\beta$-TG in CTADstabilized plasma served as a marker for platelet activation. Complement activation was investigated by measuring plasma concentrations of TCC in HEPES/EDTA-stabilized plasma. Zymosan A activated whole blood was used as a positive control for complement activation.

Following isolation of the blood samples, each tube was prepared for SEM analysis. Nonadherent blood components were washed away by rinsing the tubes extensively with 25 volumes of PBS solution. Next, adherent blood components were fixed by incubating the tubes overnight in $2.5 \%$ glutaraldehyde at $2-8^{\circ} \mathrm{C}$. Fixed samples were dehydrated by immersion in an ethanol series (50, 70, 80, 95, and 100\% ethanol). Following dehydration, the samples were air-dried. For each time point, three pieces of air-dried tubing with a length of $1 \mathrm{~cm}$ were cut out. The pieces were then cut lengthwise for analysis of the inner surface. This was done for all donors. Finally, the samples were sputter coated with gold and imaged with a scanning electron microscope.

\subsection{Experiments under static conditions: thrombin generation time assay}

This assay was carried out as described previously [1619]. Briefly, blood was withdrawn by venipuncture from a healthy, nonsmoking male blood donor who did not take any haemostasis-influencing medicines at least 10 days before the experiment. The blood was immediately anticoagulated with citrate stock solution (1 part citrate stock solution and 9 parts whole blood) and kept at $37^{\circ} \mathrm{C}$ until the start of the experiment. Uncoated, heparin-coated and PMEAcoated PVC tubes were cut to a length of $5.5 \mathrm{~cm}$. These pieces were closed at one end with a tube clamp. At the start of the experiment, blood was recalcified with $40 \mu \mathrm{L} \mathrm{CaCl}_{2}$ stock solution per $\mathrm{mL}$ blood. Subsequently, $750 \mu \mathrm{L}$ of blood was added to each tube sample and the tube samples were incubated at $37^{\circ} \mathrm{C}$ under static conditions. After 5 minutes of incubation, $17.5 \mu \mathrm{L}$ of blood was taken from each tube sample and mixed with $282.5 \mu \mathrm{L}$ stop buffer. Sampling was done 
every 5 minutes until 24 minutes of incubation, from then samples were taken every 2 minutes. Blood samples were kept on ice until further handling. At the end of the experiment, the blood samples were centrifuged at $10621 \mathrm{~g}$ for $5 \mathrm{~min}$ utes. Next, $200 \mu$ L plasma of each sample was loaded onto a microtiter plate that was kept on ice. After loading, the microtiter plate was heated for 5 minutes at $37^{\circ} \mathrm{C}$. Finally, thrombin concentrations were measured using absorption spectrophotometry at $405 \mathrm{~nm}$.

\subsection{Statistics}

Statistical analysis was performed using Mann-Whitney analysis for between-group comparisons and the Wilcoxon test for paired observations for comparison within groups. A $P$-value less than .05 (two-tailed) was considered significant.

\section{RESULTS AND DISCUSSION}

\subsection{Analysis of the blood samples}

\subsubsection{Platelet counts}

Figure 1 compiles the results on the platelet count experiments; note that data referring to the three different biomaterials, based on blood from four different donors and measured at five time points, are combined. This format is used consistently throughout this article. At the start of the experiment, the donors had platelet counts between 75,000 and 180,000 per $\mu \mathrm{L}$, which is within the normal range. Some increased spreading is noted, especially in the counts that were measured after 225 or 300 minutes. The heparin-coated specimens and the uncoated controls show invariant platelet counts as a function of time. The PMEA coating, on the other hand, induces a decrease in the concentration of circulating platelets. For donor JB, this effect can be noticed already after $75 \mathrm{~min}$, and the decrease goes on during the entire experiment. For the other donors, platelet counts start to decline only after 150 minutes (KS and SB), or after 225 minutes (WW). The largest drop in platelet count was found with the blood from donor JB in the PMEA-coated tube; the concentration of circulating platelets decreased by almost $60 \%$, from ca. 140,000 per $\mu \mathrm{L}$ to ca. 60,000 per $\mu \mathrm{L}$. At the end of the experiment, the concentration of circulating platelets was significantly lower in the PMEA-coated tubes compared to the heparin-coated tubes $(79,917 \pm 6,304$ platelets $/ \mu \mathrm{L}$ blood for PMEA-coated tubes versus $135,333 \pm 10,623$ platelets $/ \mu \mathrm{L}$ blood for heparin-coated tubes (mean \pm sem), see Table 1 (supplementary material), $P=.001$ ) and the uncoated controls $(79,917 \pm 6,304$ platelets $/ \mu \mathrm{L}$ blood for PMEA-coated tubes versus $133,833 \pm 10,650$ platelets $/ \mu \mathrm{L}$ blood for uncoated controls (mean \pm sem), see Table 1 (supplementary material), $P=.001$ ). This indicated that the PMEA coating has a propensity to activate contacting platelets. This is, most probably, not a direct effect, but an effect of plasma proteins adsorbed onto the PMEA surface. Activated platelets are known to adhere to adsorbed fibrinogen, von Willebrand factor, vitronectin, and fibronectin [20]. Consequently, this results in lower platelet counts. In addition, activated platelets adhere to each other via fibrinogen bridges,

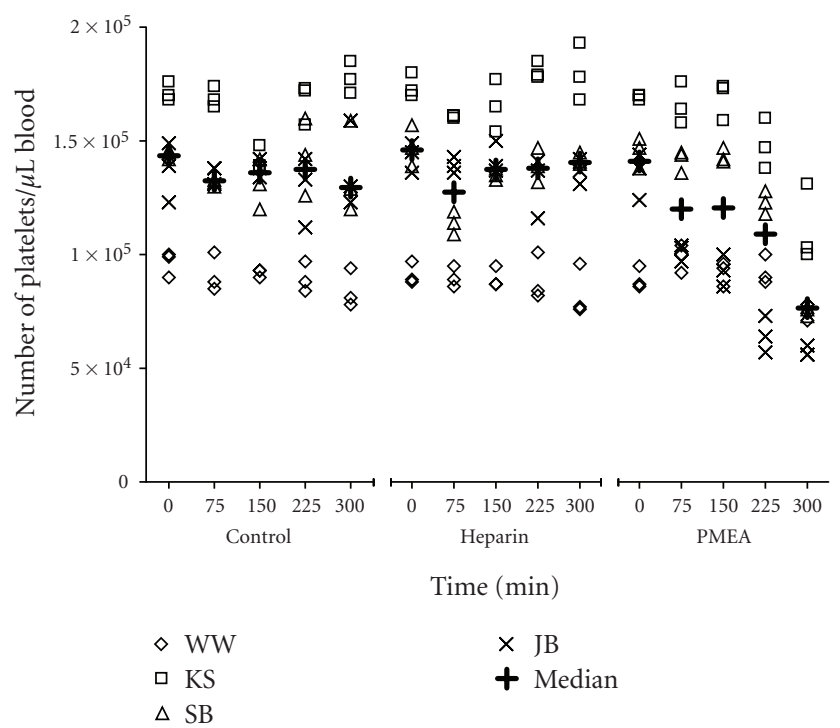

FIGURE 1: Platelet counts for the individual donors. Data are shown at five time points for each tubing surface tested. Measurements were performed in triplicate.

anchored by GPIIb/IIIa receptors [21]. Activated platelets also adhere to circulating leukocytes $[22,23]$. Some of the surface-attached platelets partially detach, leaving adherent membrane fragments [24]. Other platelets are damaged by shear forces [25]. Recently, platelet counts were also used in a clinical study to evaluate hemocompatibility of heparinand PMEA-coated CPB circuits. Kutay et al. reported a more significant depletion of circulating platelets in PMEA-coated circuits compared to heparin-coated circuits [26].

\subsubsection{Hemolysis}

Figure 2 shows the dataset resulting from our concentration measurements of extracellular (free) Hb. Rupture of erythrocytes (hemolysis) (e.g., due to collisions with the lumen of the tubes) leads to release of $\mathrm{Hb}$ into the plasma. The vertical axis in Figure 2 depicts the ratio free $\mathrm{Hb}$ : total $\mathrm{Hb}$, expressed as a percentage. Clearly, hemolysis is very low, that is, most of the erythrocytes remained intact in all cases. Even in the most extreme situation (donor JB, PMEA coating, 300 minutes circulation), only 1 out of every 400 molecules $\mathrm{Hb}$ is free. Despite the occurrence of little hemolysis, it is clear that the level of free $\mathrm{Hb}$ in the plasma increases with circulation time, which was expected. The three coatings do not perform differently in this respect. No significant differences in the levels of plasma-free $\mathrm{Hb}$ were found between the different tubes. The hemolysis assay applied in this study was based on the Cripps method to measure levels of plasma-free $\mathrm{Hb}$ [15]. In a study by Malinauskas [27], this method was shown to be more precise and accurate than the chemical addition methods to measure levels of plasma-free $\mathrm{Hb}$.

\subsubsection{Activation of platelets: $\beta$-thromboglobulin}

Figure 3 provides an overview of the concentration measurements of $\beta$-TG, which is a soluble marker for platelet 


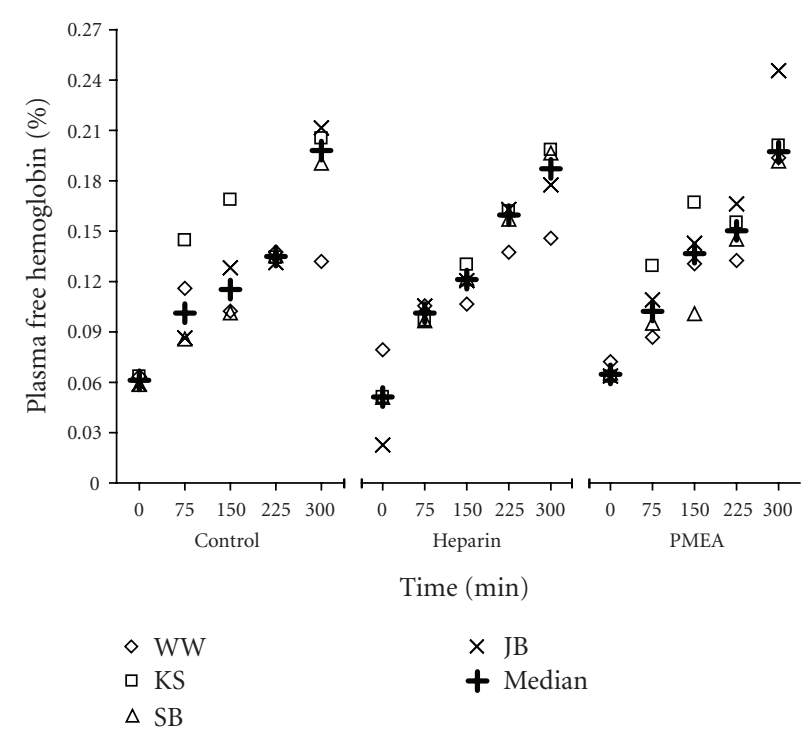

Figure 2: Percentage of plasma-free $\mathrm{Hb}$ for the individual donors. Data are shown at five time points for each tubing surface tested.

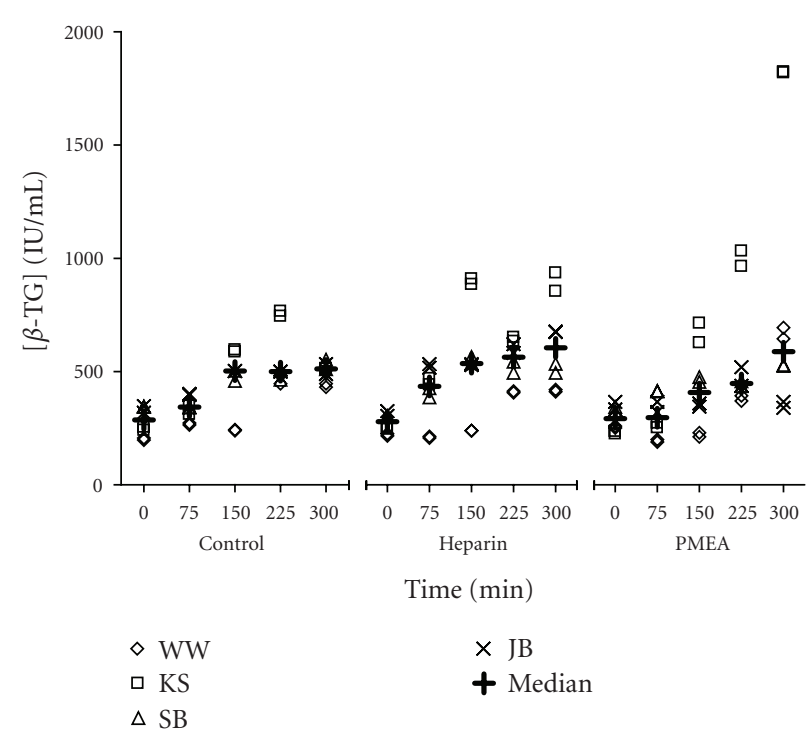

FIgURE 3: Concentrations of $\beta$-thromboglobulin for the individual donors. Data are shown at five time points for each tubing surface tested. Measurements were performed in duplicate.

activation [28]. Activated platelets release $\beta$-TG from their $\alpha$-granules.

The $\beta$-TG concentration versus time profiles show an increasing trend, revealing that platelet activation progresses with time. With the blood of the donors WW and SB, this is found consistently for all coatings. The blood of donor JB follows this pattern, except in contact with PMEA: the concentration of $\beta$-TG then remains virtually unchanged. This is remarkable, since the platelet counts for this donor and this coating were found to decrease sharply (see Figure 1). Most likely, platelets of this donor adhere to PMEA without subsequent release of the granular contents. Donor KS is slightly aberrant. The levels of $\beta$-TG are relatively high, throughout all experiments, but it must be kept in mind that this donor also had the highest platelet counts (see Figure 1). In contact with the PMEA coating, a steep increase of the concentration of $\beta$-TG is found after 150 minutes of incubation. However, there were no significant differences in platelet activation levels between uncoated, heparin-coated, and PMEAcoated tubes. This indicates that immobilization of heparin or coating with PMEA does not help to reduce platelet activation within blood that contacts a PVC surface.

\subsubsection{Activation of leukocytes: myeloperoxidase}

During ECC, contact between the blood and artificial surfaces is known to induce an inflammatory response characterized by the activation of various leukocyte cell types $[23,29]$. Of the various leukocytes, neutrophils are the most abundant; they play a central role in the inflammatory response to CPB [27]. MPO is a glycoprotein abundantly present in the primary granules of neutrophils; activated neutrophils release MPO by degranulation [23, 30, 31].

Figure 4 presents the data of the concentration measurements of MPO. A clear pattern emerging from the data is that for every donor, the concentration of MPO increases continuously. The rise in MPO levels was the highest in PMEA-coated tubes. This resulted in significantly higher levels of MPO compared to heparin-coated tubes, at time points 225 minute $(163 \pm 11.6 \mathrm{ng} / \mathrm{mL}$ for PMEA-coated tubes versus $131.5 \pm 4.8 \mathrm{ng} / \mathrm{mL}$ for heparin-coated tubes (mean \pm sem), see Table 1 (supplementary material), $P=.01$ ) and 300 minute $(222.3 \pm 11.5 \mathrm{ng} / \mathrm{mL}$ for PMEA-coated tubes versus $157.7 \pm 3.6 \mathrm{ng} / \mathrm{mL}$ for heparin-coated tubes (mean \pm sem), see Table 1 (supplementary material), $P=.001$ ), and to uncoated controls, at time point 300 minute $(222.3 \pm$ $11.5 \mathrm{ng} / \mathrm{mL}$ for PMEA-coated tubes versus $176.8 \pm 9.8 \mathrm{ng} / \mathrm{mL}$ for uncoated controls (mean \pm sem), see Table 1 (supplementary material), $P=.01$ ). These data indicate that the PMEA coating induces MPO release more abundantly as compared to both other surfaces. It is of interest to compare our data with a recent study of Lappegård et al. who also used the Chandler loop model to investigate neutrophil activation after blood-artificial surface contact [23,29]. After 4 hours of blood circulation they found significantly lower levels of MPO release in heparin-coated tubes compared to uncoated controls. Lappegård et al., however, used a heparin coating based on covalently end point-attached heparin [29], while the heparin coating used in our experiments involved both covalent and ionic interactions of heparin with the surface.

Our results appear to be in line with a recent clinical study by Kutay et al. who compared the hemocompatibility of PMEA- and heparin-coated CPB [26]. MPO levels at the end of $\mathrm{CPB}$ were significantly higher in the PMEA-coated circuits compared to the heparin-coated circuits. Besides MPO, plasma levels of interleukin-8, a proinflammatory cytokine, were also quantified by ELISA. In the three different tubes, plasma levels of interleukin-8 remained undetectable until 225 minutes of incubation. At the end of the experiment, interleukin-8 generation did not differ significantly between the three tubes and plasma levels never exceeded $90 \mathrm{pg} / \mathrm{mL}$ (data not shown). Apparently, 5 hours of blood-biomaterial 


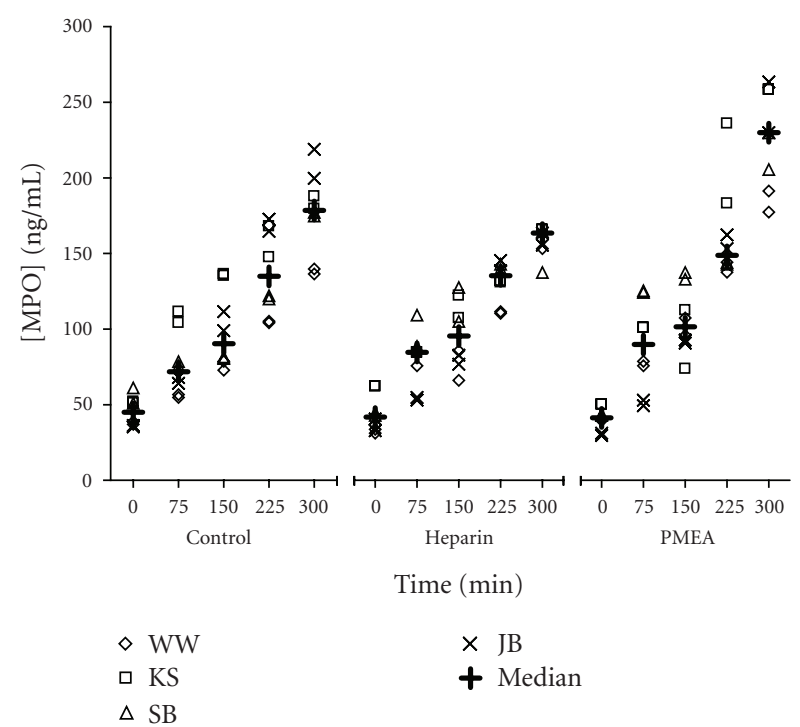

Figure 4: Concentrations of myeloperoxidase for the individual donors. Data are shown at five time points for each tubing surface tested. Measurements were performed in duplicate.

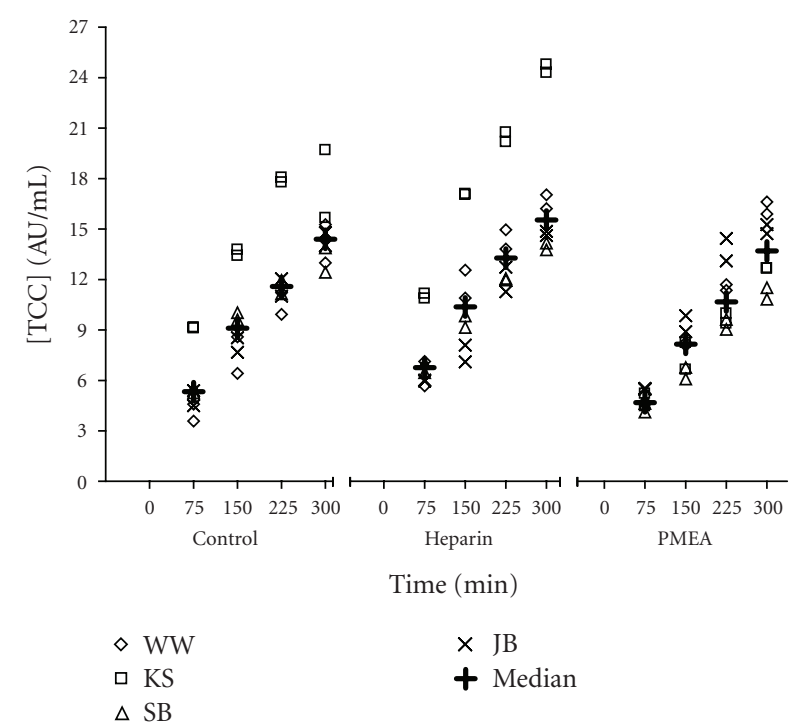

Figure 5: Concentrations of terminal complement complex for the individual donors. Data are shown at five time points for each tubing surface tested. Measurements were performed in duplicate. As can be seen, TCC was undetectable in all samples at time point 0 hour.

contact in our model are not sufficient to use interleukin-8 as a discriminating marker for inflammatory responses.

\subsubsection{Activation of the complement system: terminal complement complex}

Complement activation has been studied extensively as a marker for hemocompatibility of artificial surfaces. Different components of the complement activation cascade can be used [31]. However, in a study by Gong et al. [32], gen- eration of complement activation products in the Chandler loop model displayed component-specific responsiveness to the size of the gas surface and the biomaterial surface. Of the complement activation markers evaluated, generation of TCC was least influenced by the size of the gas surface and mainly dependent on the biomaterial surface. This prompted us to use TCC as a complement activation marker in our study. Figure 5 depicts the data of the concentration measurements of TCC. The levels of TCC were undetectable at the start of the experiments but increased steadily in all tubes throughout the experiment. At several time points, significantly higher levels of TCC could be detected in heparin-coated tubes compared to PMEA-coated tubes (at 75 minute: $7.5 \pm 0.8 \mathrm{AU} / \mathrm{mL}$ for heparin-coated tubes versus $4.8 \pm 0.2 \mathrm{AU} / \mathrm{mL}$ for PMEA-coated tubes (mean \pm sem), see Table 1 (supplementary material), $P=.001$; at 150 minute: $11.5 \pm 1.4 \mathrm{AU} / \mathrm{mL}$ for heparin-coated tubes versus $7.9 \pm 0.4 \mathrm{AU} / \mathrm{mL}$ for PMEA-coated tubes (mean \pm sem), see Table 1 (supplementary material), $P=.024$; at 225 minute: $14.7 \pm 1.3 \mathrm{AU} / \mathrm{mL}$ for heparin-coated tubes versus $11.1 \pm$ $0.7 \mathrm{AU} / \mathrm{mL}$ for PMEA-coated tubes (mean \pm sem), see Table 1 (supplementary material), $P=.027$ ) and uncoated controls (at 75 minute: $7.5 \pm 0.8 \mathrm{AU} / \mathrm{mL}$ for heparin-coated tubes versus $5.9 \pm 0.7 \mathrm{AU} / \mathrm{mL}$ for uncoated controls (mean $\pm \mathrm{sem}$ ), see Table 1 (supplementary material), $P=.036$ ). This suggests that TCC generation proceeds faster in heparin-coated tubes compared to PMEA-coated tubes and uncoated controls. Several in vitro studies using the Chandler loop model reported prevention of TCC generation by heparin-coated PVC compared to uncoated PVC [20, 23, 29, 33]. However, none of these studies had blood circulation times of more than two hours. Also, these studies used heparin coatings which were structurally different from the heparin coating evaluated in our study. Weber et al. compared covalently heparin-coated tubes from four different manufacturers and also found marked differences in hemocompatibility [11].

\subsection{Analysis of the inner surface}

\subsubsection{Scanning electron microscopy}

A set of scanning electron micrographs was recorded (3 different materials, 4 donors, 5 different times of circulation in the Chandler loop system, and three samples of every tube were examined). In general, we observed that adhesion of blood components developed slowly and gradually as the experiments proceeded. However, the SEM data revealed a striking difference between uncoated PVC and heparincoated PVC on one hand, and the PMEA-coated PVC on the other hand. Four micrographs, taken after 5 hours of blood circulation over the surfaces, are shown in Figure 6 to illustrate this difference. The uncoated PVC and heparin-coated PVC surfaces showed a remarkable resemblance. These surfaces were, to an extent of approximately $80 \%$, devoid of any visible adherent blood components. There were, however, island-like regions, usually small (e.g., $10 \times 10 \mu \mathrm{m}$ ) but sometimes larger (e.g., $100 \times 200 \mu \mathrm{m})$. Enlarged images of these islands (see Figures 6(a) and 6(b)) revealed a flat patch-like structure, presumably composed of fibrin 


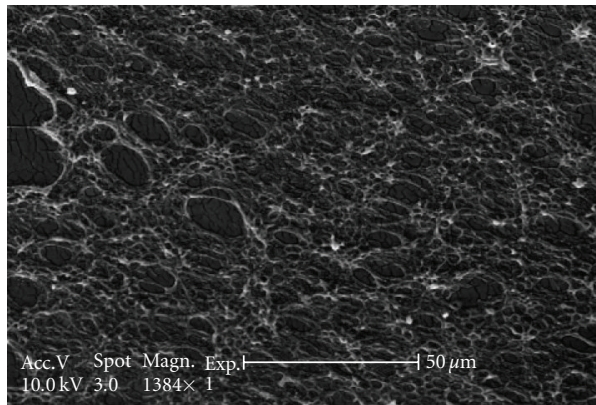

(a)

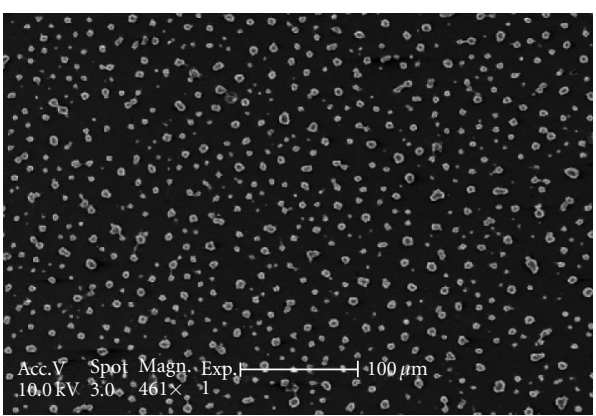

(c)

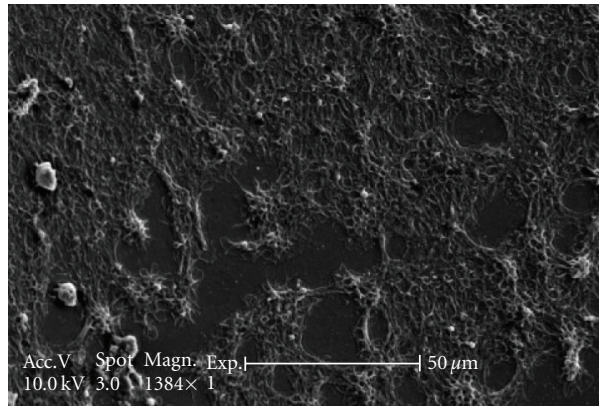

(b)

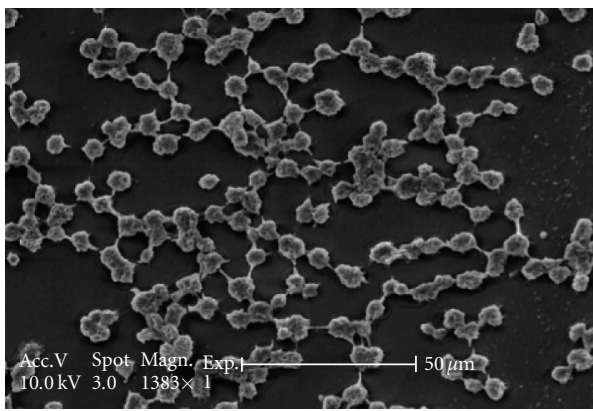

(d)

FIGURE 6: (a) Scanning electron micrograph of a typical island structure on the inner surface of the uncoated (control) tube, after 5 hours contact with full human blood in the Chandler loop model. A patch-like structure is seen, which is, presumably, composed of fibrin threads, which adhered to the surface. (b) Scanning electron micrograph of a typical island structure on the inner surface of the heparin-coated tube (same conditions). The patch-like structures in (a) and (b) are similar; note that platelets as well as cells (presumably leukocytes) are seen in (b). (c) Scanning electron micrograph of the PMEA-coated surface, after 5 hours of experimentation in the Chandler loop. Adhered cells are observed, either individually, or in small aggregates of 2 or 3 cells. Cells and aggregates are, to a good approximation, evenly spread over the surface, although regions of relatively high cell density could be discerned. (d) Close-up on a region of relatively high cell density. Note that most of the adherent cells are engaged into cell-cell contacts through pseudopodia.

threads. Some blood platelets were entrapped in the patch, in the case of the uncoated surface (see Figure 6(a)). For the heparin-coated surface, platelets as well as larger cells (presumably leukocytes) were entrapped in or adhered to the patch structure (see Figure 6(b)). Evaluation of the inner surface of the PMEA-coated tubing showed radically different pictures as can be seen in Figures 6(c) and 6(d). Fibrin formation is evident on the uncoated and heparin-coated surfaces, but not for the PMEA-coating. Scattered over the PMEA-coated surface, we encountered isolated cells, or ensembles of a small number of cells (typically 2 or 3 cells). Presumably, the adhered cells are leukocytes; the diameter of these cells is $10-15 \mu \mathrm{m}$. Figure 6(d) shows a detailed SEM micrograph of a region that was relatively densely populated with adherent cells. It is seen that most of the cells extend pseudopodia, through which they are connected to one or more neighbors. Leukocyte activation induced by surface contact, leading to pseudopodia (see Figure 6(d)), can also explain why the MPO concentration was highest for the PMEA-coated PVC tube as compared to the other two tubes (see Figure 4).

\subsubsection{Measurement of thrombin generation times}

In view of the differences between these coatings encountered after prolonged blood contact in the Chandler loop, we de- cided to subject the surfaces also to the well-known thrombin generation assay [16-19]. Contact activation of the blood coagulation system is accompanied by a sudden increase of thrombin levels, after a lag-time that varies between approximately 5 minutes for highly thrombogenic materials to approximately 60 minutes for materials with extremely low thrombogenicity. Figure 7 shows the thrombin generation curves measured in triplicate with the uncoated, heparincoated, and PMEA-coated PVC tubes. Thrombin generation occurred between 30 and 40 minutes in the uncoated PVC tubes. Coating of the PVC with immobilized heparin prolonged the thrombin generation time until 60 minutes. This clearly indicates the better antithrombogenic properties of the heparin-coated PVC tubes compared to uncoated PVC tubes. A remarkable finding, however, was that coating of PVC tubes with PMEA resulted in thrombin generation times comparable to those of the uncoated PVC tubes. Note that, in the case of the heparin-coated PVC, the thrombin concentration remains $<2 \mathrm{nM}$, even after 60 minutes. The analysis was stopped after this time point since the assay is static. The red blood cells may show aberrant behavior after 1 hour of static conditions (Rouleaux formation), which compromises the reliability of the method. Comparing the thrombin generation curves, we conclude that thrombogenicity of the three different surfaces is as follows: uncoated PVC $\approx$ PMEA-coated PVC $>$ heparin-coated PVC. 


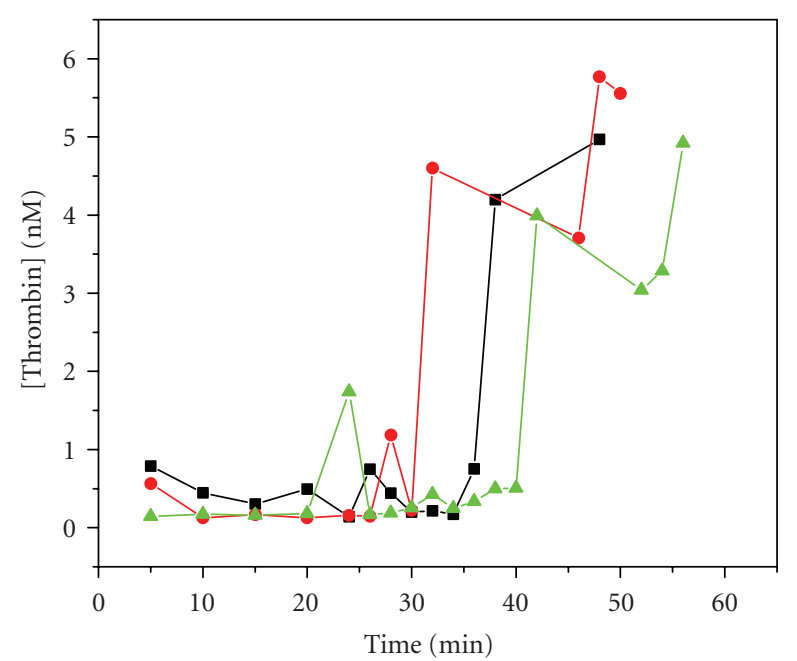

(a)

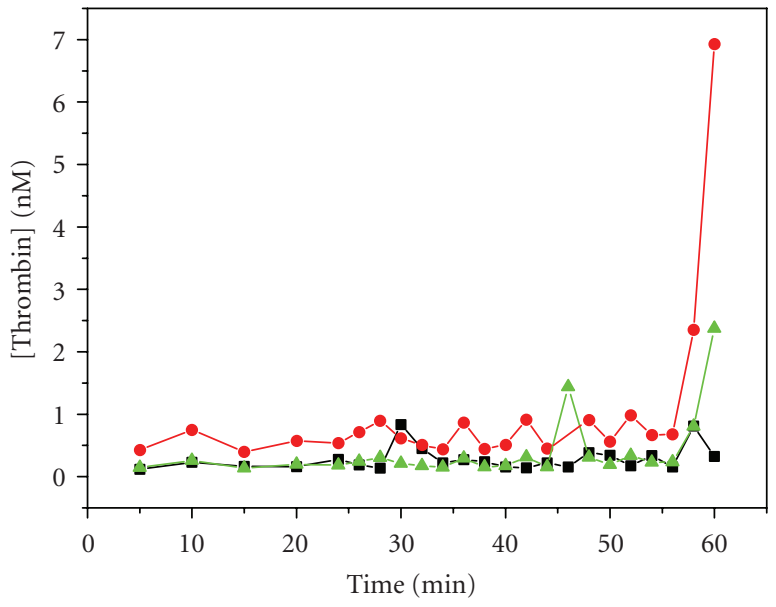

(b)

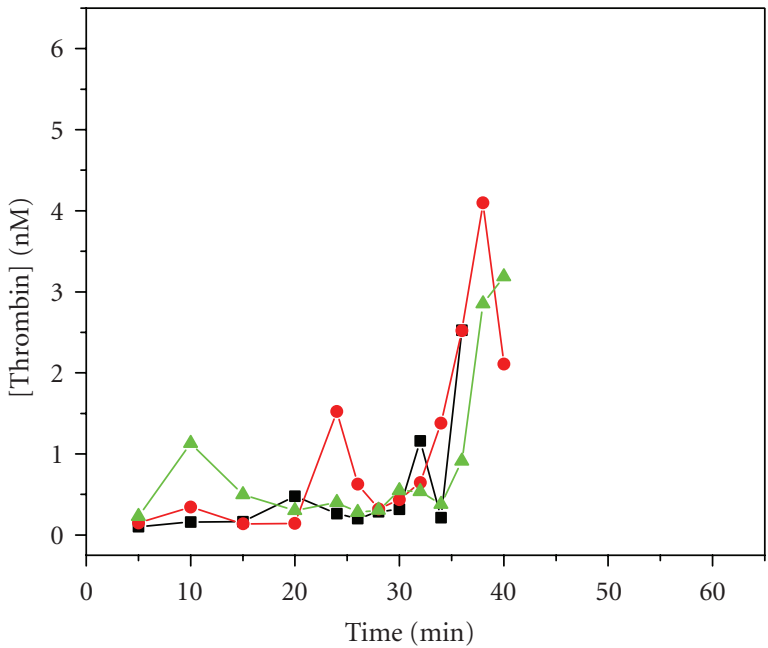

(c)

FIGURE 7: Thrombin generation curves measured for uncoated PVC (a), heparin-coated PVC (b), and PMEA-coated PVC (c) incubated with recalcified blood under static conditions. Experiments were performed in triplicate, that is, every blood sample was analyzed three times.

\subsubsection{The Chandler loop model}

After collecting all data, we wondered whether any correlation could be defined between the intensity of bloodbiomaterial contact in the Chandler loop system, and that in real-life extracorporeal circulation in CPB. Assuming that the level of blood-biomaterial contact $(Q)$ is proportional to the surface area of the artificial material $(S)$ and the time of blood-biomaterial contact $(t)$, and inversely proportional to the blood volume $(V)$, it can be calculated for the Chandler loop system (with $S=40 \mathrm{~cm}^{2}, V=7 \mathrm{~mL}, t=5 \mathrm{~h}$ ), that $Q=$ $28.6 \mathrm{~h} / \mathrm{cm}$. For a typical CPB system (with $S=25,000 \mathrm{~cm}^{2}, V$ $=8,000 \mathrm{~mL}(6 \mathrm{~L}$ of blood $+2 \mathrm{~L}$ of priming fluid $))$, it then follows that $t=(28.6 \times 8,000) / 25,000 \approx 9 \mathrm{~h}$. In other words, this simple model indicates that 5 hours of experimentation in the Chandler loop corresponds with a level of bloodbiomaterial contact that corresponds to at least 9 hours of operation in a typical CPB system. Most probably, the estimated 9 hours is an underestimation, since the blood cells in real-life CPB are oxygenated and the blood resides mostly within the patient's vasculature. This probably implies that some recuperation of blood cells occurs during real-life CPB, while this is evidently not the case in our model system. Noteworthy, the extent of hemolysis encountered in our experiments was very low, that is, $<0.3 \%$ after 5 hours of circulation, irrespective of the donor or (coated) surface.

\section{CONCLUSION}

Systematic evaluation of the blood biomaterial contact for the three different tubings, using relatively long periods of blood-biomaterial contact, was performed. The three different surfaces were as follows: uncoated PVC, heparincoated PVC, and PMEA-coated PVC; the latter two are already in clinical use, as tubings in extracoporeal circulation equipment. Clear differences with respect to platelet counts, leukocyte activation (MPO release), and deposition of blood components at the inner surfaces were found. Most 
of these differences became apparent only after the first 23 hours of experimentation. This underlines the importance of extended evaluation of blood-contacting biomaterials that are to be used in long-term applications, such as extended $\mathrm{CPB}$. It is noteworthy that the PMEA-coated tubes showed a relatively low level of hemocompatibility. Compared with uncoated PVC and heparin-coated PVC, (i) a substantial drop of the platelet counts, (ii) activation of leukocytes and marked adherence of leukocytes at the inner surface, and (iii) a thrombogenicity comparable to uncoated PVC were observed. The present results lead to two important conclusions.

(1) The Chandler loop system is the most useful method for the evaluation of blood-biomaterial interactions, which is most probably relevant for the development of equipment for extracorporeal circulation. Extended experimentation times (e.g., 5 hours) are mandatory, since differences for various materials may masquerade during the first few hours.

(2) PMEA biomaterial is probably not optimal for use in extracorporeal circulation equipment; further improvements are necessary.

\section{ACKNOWLEDGMENTS}

The help of H. Jussen and F. Spee (Instrument Development Engineering and Evaluation, University of Maastricht) is gratefully acknowledged. This study was financed within the framework of the Bioterials program, a 4-years privatepublic joint effort of the Dutch Ministry of Economic Affairs, the Province of Dutch Limburg, DSM Research BV (Geleen, the Netherlands), the Academic Hospital Maastricht, and the University of Maastricht. Bioterials runs in the period April 2005-April 2009.

\section{REFERENCES}

[1] S. C. Skinner, R. B. Hirschl, and R. H. Bartlett, "Extracorporeal life support," Seminars in Pediatric Surgery, vol. 15, no. 4, pp. 242-250, 2006.

[2] O. Mangoush, S. Purkayastha, S. Haj-Yahia, et al., "Heparinbonded circuits versus nonheparin-bonded circuits: an evaluation of their effect on clinical outcomes," European Journal of Cardio-Thoracic Surgery, vol. 31, no. 6, pp. 1058-1069, 2007.

[3] F. D. Rubens, "Cardiopulmonary bypass technology transfer: musings of a cardiac surgeon," Journal of Biomaterials Science, Polymer Edition, vol. 13, no. 4, pp. 485-499, 2002.

[4] H. P. Wendel and G. Ziemer, "Coating-techniques to improve the hemocompatibility of artificial devices used for extracorporeal circulation," European Journal of Cardio-Thoracic Surgery, vol. 16, no. 3, pp. 342-350, 1999.

[5] D. Baykut, F. Bernet, J. Wehrle, K. Weichelt, P. Schwartz, and H. R. Zerkowski, "New surface biopolymers for oxygenators: an in vitro hemocompatibility test of poly(2methoxyethylacrylate)," European Journal of Medical Research, vol. 6, no. 7, pp. 297-305, 2001.

[6] X. M. Mueller, D. Jegger, M. Augustburger, J. Horisberger, and L. K. von Segesser, "Poly 2-methoxyethylacrylate (PMEA) coated oxygenator: an ex vivo study," International Journal of Artificial Organs, vol. 25, no. 3, pp. 223-229, 2002.
[7] M. Ninomiya, K. Miyaji, and S. Takamoto, "Influence of PMEA-coated bypass circuits on perioperative inflammatory response," Annals of Thoracic Surgery, vol. 75, no. 3, pp. 913917, 2003.

[8] J. O’Neill, G. Schutze, M. Heulitt, P. Simpson, and B. Taylor, "Nosocomial infections during extracorporeal membrane oxygenation," Intensive Care Medicine, vol. 27, no. 8, pp. 12471253, 2001.

[9] D. P. Mason, D. J. Boffa, S. C. Murthy, et al., "Extended use of extracorporeal membrane oxygenation after lung transplantation," Journal of Thoracic and Cardiovascular Surgery, vol. 132, no. 4, pp. 954-960, 2006.

[10] K. Sung, Y. T. Lee, P. W. Park, et al., "Improved survival after cardiac arrest using emergent autopriming percutaneous cardiopulmonary support," Annals of Thoracic Surgery, vol. 82, no. 2, pp. 651-656, 2006.

[11] N. Weber, H. P. Wendel, and G. Ziemer, "Quality assessment of heparin coatings by their binding capacities of coagulation and complement enzymes," Journal of Biomaterials Applications, vol. 15, no. 1, pp. 8-22, 2000.

[12] D. S. Rijkers, S. J. H. Wielders, G. I. Tesser, and H. C. Hemker, "Design and synthesis of thrombin substrates with modified kinetic parameters," Thrombosis Research, vol. 79, no. 5-6, pp. 491-499, 1995.

[13] A. B. Chandler, "In vitro thrombotic coagulation of the blood; a method for producing a thrombus," Laboratory Investigation, vol. 7, no. 2, pp. 110-114, 1958.

[14] R. Kopp, R. Bensberg, A. Kashefi, K. Mottaghy, R. Rossaint, and R. Kuhlen, "Effect of hirudin versus heparin on hemocompatibility of blood contacting biomaterials: an in vitro study," International Journal of Artificial Organs, vol. 28, no. 12, pp. 1272-1277, 2005.

[15] C. M. Cripps, "Rapid method for the estimation of plasma haemoglobin levels," Journal of Clinical Pathology, vol. 21, no. 1, pp. 110-112, 1968.

[16] Y. B. J. Aldenhoff, M. L. W. Knetsch, J. H. L. Hanssen, T. Lindhout, S. J. H. Wielders, and L. H. Koole, "Coils and tubes releasing heparin. Studies on a new vascular graft prototype," Biomaterials, vol. 25, no. 16, pp. 3125-3133, 2004.

[17] Y. B. J. Aldenhoff, R. Blezer, T. Lindhout, and L. H. Koole, "Photo-immobilisation of dipyridamole (Persantin ${ }^{\circledR}$ ) at the surface of polyurethane biomaterials: reduction of in-vitro thrombogenicity," Biomaterials, vol. 18, no. 2, pp. 167-172, 1997.

[18] T. W. Barrowcliffe, M. Cattaneo, G. M. Podda, et al., "New approaches for measuring coagulation," Haemophilia, vol. 12, supplement 3, pp. 76-81, 2006.

[19] W. van Oeveren, J. Haan, P. Lagerman, and P. Schoen, "Comparison of coagulation activity tests in vitro for selected biomaterials," Artificial Organs, vol. 26, no. 6, pp. 506-511, 2002.

[20] N. Weber, H. P. Wendel, and G. Ziemer, "Hemocompatibility of heparin-coated surfaces and the role of selective plasma protein adsorption," Biomaterials, vol. 23, no. 2, pp. 429-439, 2002.

[21] G. M. Sreeram, A. D. Sharma, and T. F. Slaughter, "Platelet glycoprotein IIb/IIIa antagonists: perioperative implications," Journal of Cardiothoracic and Vascular Anesthesia, vol. 15, no. 2, pp. 237-240, 2001.

[22] C. S. Rinder, J. L. Bonan, H. M. Rinder, J. Mathew, R. Hines, and B. R. Smith, "Cardiopulmonary bypass induces leukocyteplatelet adhesion,” Blood, vol. 79, no. 5, pp. 1201-1205, 1992. 
[23] K. T. Lappegård, M. Fung, G. Bergseth, et al., "Effect of complement inhibition and heparin coating on artificial surfaceinduced leukocyte and platelet activation," Annals of Thoracic Surgery, vol. 77, no. 3, pp. 932-941, 2004.

[24] Y.-P. Wu, P. G. de Groot, and J. J. Sixma, "Shear stress-induced detachment of blood platelets from various surfaces," Arteriosclerosis, Thrombosis, and Vascular Biology, vol. 17, no. 11, pp. 3202-3207, 1997.

[25] K. Kawahito, J. Mohara, Y. Misawa, and K. Fuse, "Platelet damage caused by the centrifugal pump: in vitro evaluation by measuring the release of $\alpha$-granule packing proteins," Artificial Organs, vol. 21, no. 10, pp. 1105-1109, 1997.

[26] V. Kutay, T. Noyan, S. Ozcan, Y. Melek, H. Ekim, and C. Yakut, "Biocompatibility of heparin-coated cardiopulmonary bypass circuits in coronary patients with left ventricular dysfunction is superior to PMEA-coated circuits," Journal of Cardiac Surgery, vol. 21, no. 6, pp. 572-577, 2006.

[27] R. A. Malinauskas, "Plasma hemoglobin measurement techniques for the in vitro evaluation of blood damage caused by medical devices," Artificial Organs, vol. 21, no. 12, pp. 12551267, 1997.

[28] R. Ohkawa, Y. Hirowatari, K. Nakamura, et al., "Platelet release of $\beta$-thromboglobulin and platelet factor 4 and serotonin in plasma samples," Clinical Biochemistry, vol. 38, no. 11, pp. 1023-1026, 2005.

[29] K. T. Lappegård, M. Fung, G. Bergseth, J. Riesenfeld, and T. E. Mollnes, "Artificial surface-induced cytokine synthesis: effect of heparin coating and complement inhibition," Annals of Thoracic Surgery, vol. 78, no. 1, pp. 38-44, 2004.

[30] A. E. Åsberg and V. Videm, "Neutrophil dysfunction after biomaterial contact in an in vitro model of cardiopulmonary bypass," European Journal of Cardio-Thoracic Surgery, vol. 30, no. 5, pp. 744-748, 2006.

[31] A. E. Åsberg and V. Videm, "Activation of neutrophil granulocytes in an in vitro model of a cardiopulmonary bypass," Artificial Organs, vol. 29, no. 12, pp. 927-936, 2005.

[32] J. Gong, R. Larsson, K. N. Ekdahl, T. E. Mollnes, U. Nilsson, and B. Nilsson, "Tubing loops as a model for cardiopulmonary bypass circuits: both the biomaterial and the blood-gas phase interfaces induce complement activation in an in vitro model," Journal of Clinical Immunology, vol. 16, no. 4, pp. 222-229, 1996.

[33] J. Andersson, J. Sanchez, K. N. Ekdahl, G. Elgue, B. Nilsson, and R. Larsson, "Optimal heparin surface concentration and antithrombin binding capacity as evaluated with human nonanticoagulated blood in vitro," Journal of Biomedical Materials Research Part A, vol. 67, no. 2, pp. 458-466, 2003. 

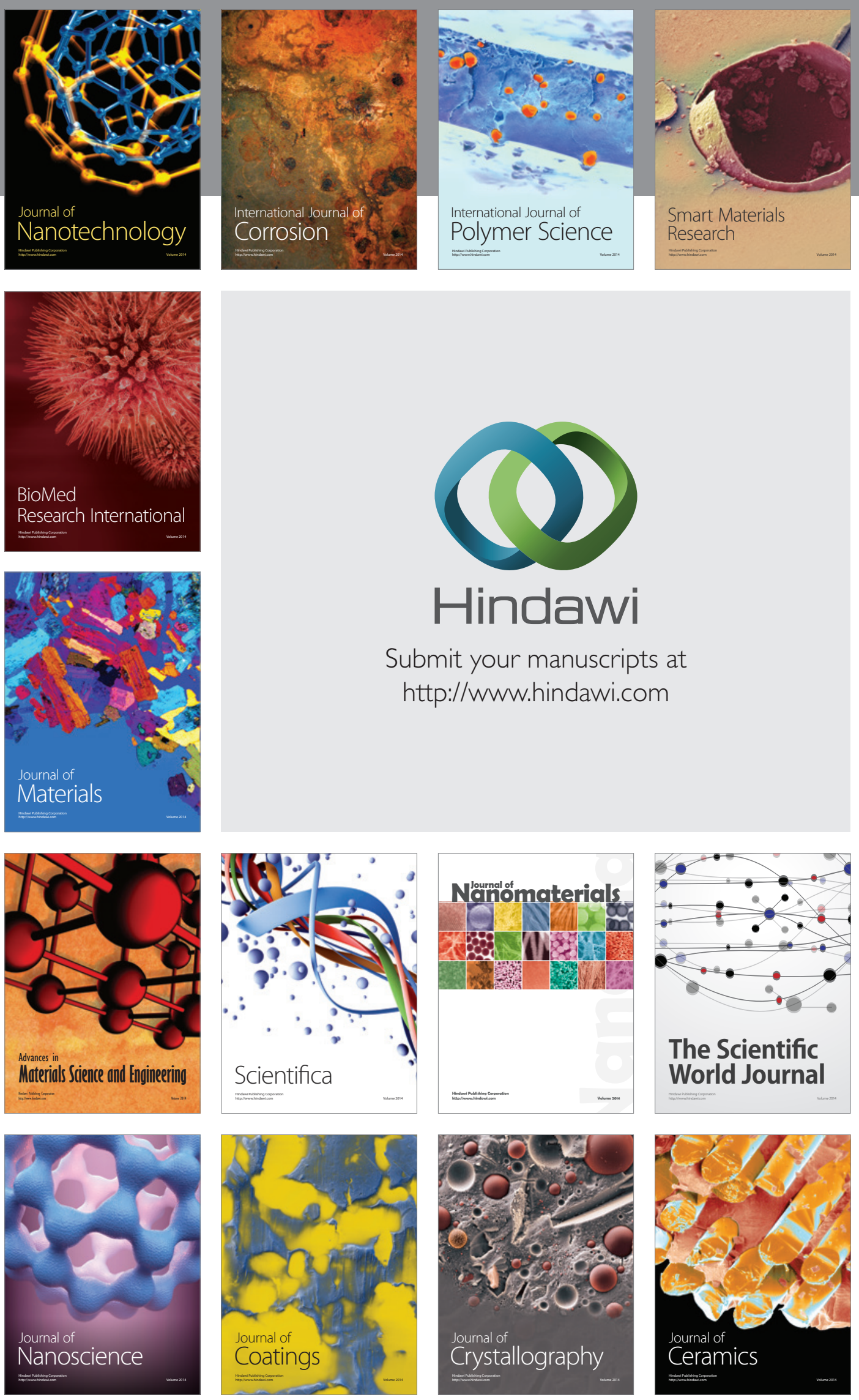

The Scientific World Journal

Submit your manuscripts at

http://www.hindawi.com

\section{World Journal}

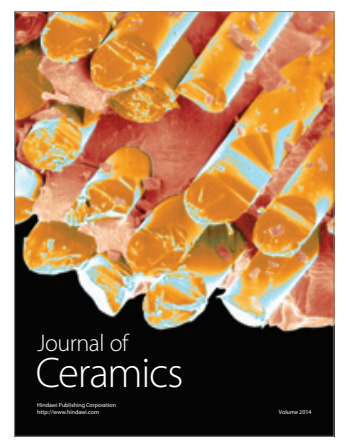

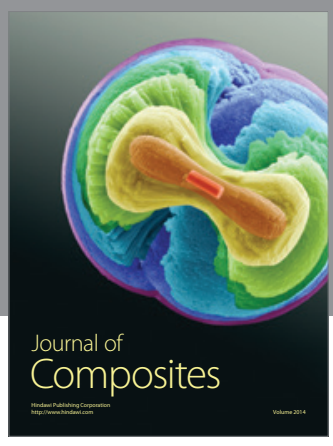
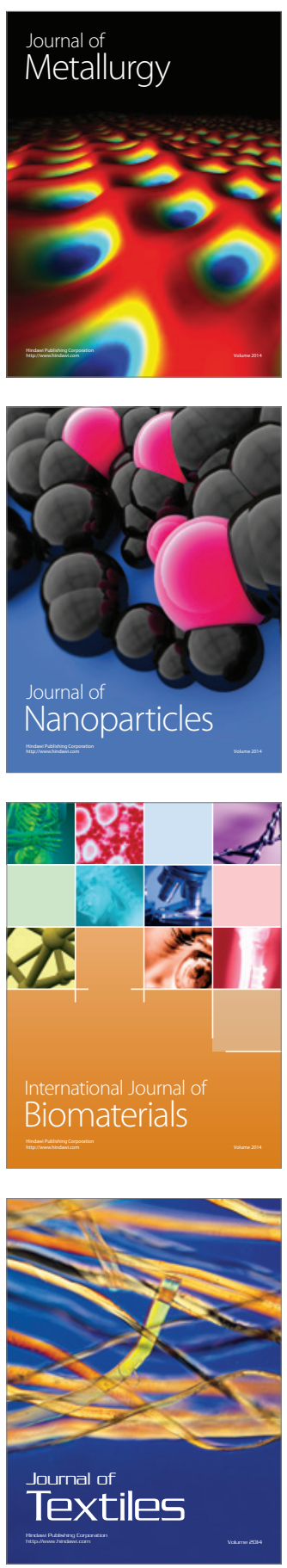